\title{
A SOLITARY GROUP OF TWO-DIMENSIONAL DEEP-WATER WAVES*
}

\author{
$\mathrm{BY}$
}

CHIA-SHUN YIH

University of Michigan

\begin{abstract}
Summary. If the magnitude of a line of concentrated force moving with a constant velocity on the surface of deep water oscillates with a constant frequency, a single group of two-dimensional gravity waves is created. The group moves with the velocity of the concentrated force, and the waves in it have a wave number and phase velocity which are determined by the velocity of the concentrated force and the frequency of oscillation of its magnitude. If these latter quantities are such that the velocity of the force is near the group velocity, in the classical sense, of the waves created, the length of the group will be very long, but in general it can take on any value. The phase velocity of the waves created is larger than that of classical deep-water waves of the same wave number, whereas the velocity of the group is less than the group velocity of these classical waves. The respective differences, as well as the magnitude of the concentrated force, tend to zero as the length of the group increases indefinitely. The solution given provides the elemental solution from which gravity wave groups caused by any travelling oscillating pressure distribution can be found.
\end{abstract}

1. Introduction. It is well known that when a time-independent two-dimensional pressure distribution, applied to the surface of a body of water, travels with a constant velocity, gravity waves are created behind and travel with it. It is easily seen that if a stationary but oscillating two-dimensional pressure distribution is applied to the water surface, gravity waves will be created on both sides of it, travelling toward infinity in opposite directions. It is then to be expected that if the oscillatory pressure distribution travels with a constant velocity on the water surface there will be gravity waves fore and aft. The solution for the ensuing flow could in principle be obtained by superposition from the solution given by Poisson (1816) and Cauchy (1827), as quoted by Lamb [1, p. 384], for instantaneous force applied to the water surface. But the calculation for the asymptotic state (for large values of time $t$ ) will be not only very involved and tedious but also unnecessary.

\footnotetext{
* Received January 2, 1986.
} 
In this paper a simple solution for gravity waves created by a concentrated oscillatory force travelling with a constant velocity on the surface of deep water is given. A single group of gravity waves is found, which moves with the same velocity as the surface force. Within it, individual waves have a wave number $k$ and a phase velocity $c$, which are determined by the velocity $c_{g}$ and the frequency $\omega$ of the surface force. As $c_{g}$ approaches the classical group velocity for waves with frequency $\omega$, the envelope of the group will be very long (the length of the group can be defined). In the limit it will be infinite and the magnitude of the force will be zero, in agreement with the classical concept of group velocity. But in general, the group can have any length; the phase velocity of the individual waves created is larger, whereas the velocity of the group is smaller, than their respective classical counterparts for the same frequency $\omega$.

2. Surface gravity waves. Surface gravity waves represent the archetype of dispersive waves, and the simplest among these are two-dimensional deep-water waves, which will therefore be treated first.

We will assume the wave motion to be irrotational, so that the velocity has a potential $\phi$ which satisfies the Laplace equation

$$
\phi_{x x}+\phi_{y y}=0
$$

in which $x$ and $y$ are Cartesian coordinates, with $x$ measured in a horizontal direction and $y$ measured in the direction opposite to the gravitational acceleration. One boundary condition is

$$
\phi \rightarrow 0 \quad \text { as } y \rightarrow-\infty .
$$

At the free surface, the kinematic condition is, in its linear form,

$$
\eta_{t}=\phi_{y},
$$

in which $\eta$ is the displacement of the free surface from its equilibrium position and $t$ denotes the time. The Bernoulli equation at the free surface is

$$
p=-\rho\left(\phi_{t}+g \eta\right)
$$

where $p$ denotes pressure, $\rho$ denotes the density, and $g$ is the gravitational acceleration. An arbitrary function of time has been omitted from (4), because it can be absorbed in the term $\phi_{t}$, and $p$ is assumed zero whenever the right-hand side of (4) is zero. Conditions (3) and (4) are imposed at $y=0$. On differentiation of (4) with respect to $t$ and use of (3), one has

$$
p_{t}=-\rho\left(\phi_{t t}+g \phi_{y}\right) .
$$

One seeks to find a solution for a single wave group, with the feature that the envelope of the group propagates with a velocity $c_{g}$ and the individual waves propagate with velocity $c$ within the envelope, the amplitude of which approaches zero as $x$ approaches $\pm \infty$.

As will become clear, the solution contains infinitely many terms. The motivation for this series solution is obscure unless one presents the solution in the way of its discovery. One tries, at first, the single-term solution

$$
\phi=a \operatorname{sech} \varepsilon\left(z-c_{g} t\right) \exp [-i k(z-c t)], \quad z=x+i y,
$$


or, to save writing,

$$
\phi=a \operatorname{sech} \varepsilon \xi e^{-i k \zeta}
$$

where

$$
\xi=z-c_{g} t, \quad \zeta=z-c t
$$

We pointed out immediately that the term $\operatorname{sech} \varepsilon \xi$ has singularities in the fluid domain. Since these can be removed without affecting the course of the calculation, we shall ignore them for the time being and discuss the matter of singularity removal in a later section. It is to be understood that the real part of (7) is meant for $\phi$. Obviously, (7) and hence its real and imaginary parts satisfy (1) and (2). Substitution of (7) into (5) gives

$$
\begin{aligned}
-p_{t} / \rho=a\{ & \varepsilon^{2} c_{g}^{2} \operatorname{sech} \varepsilon \xi\left(1-2 \operatorname{sech}^{2} \varepsilon \xi\right)-k^{2} c^{2} \operatorname{sech} \varepsilon \xi \\
& +2 i k \varepsilon c c_{g} \operatorname{sech} \varepsilon \xi \tanh \varepsilon \xi+k g \operatorname{sech} \varepsilon \xi \\
& -i \varepsilon g \operatorname{sech} \varepsilon \xi \tanh \varepsilon \xi\} e^{-i \zeta}
\end{aligned}
$$

which shows that if we take

$$
-k^{2} c^{2}+\varepsilon^{2} c_{g}^{2}+g k=0
$$

and

$$
2 k c c_{g}=g
$$

we have

$$
p_{t} / \rho=2 a \varepsilon^{2} c_{g}^{2} \operatorname{sech}^{3} \varepsilon \xi e^{-i \zeta}
$$

on the free surface. Again, the real part of the right-hand side is meant. When the pressure distribution is given by (12), we do have a single wave group travelling with velocity $c_{g}$, with the individual waves travelling with velocity $c$. These wave velocities are given by (10) and (11), or

$$
\begin{aligned}
& c^{2}=\frac{g}{k} \frac{1+\sqrt{1+(\varepsilon / k)^{2}}}{2}, \\
& c_{g}=\frac{g}{2 k c} .
\end{aligned}
$$

It is interesting that for $\varepsilon^{2} \ll k^{2}$ the $c$ given by (13) is very close to the wave velocity of classical deep-water waves with wave number $k$, and $c_{g}$ is very close to their group velocity in the classical sense. But in general $c$ is greater, whereas $c_{g}$ is smaller, than its counterpart in classical theory.

The only artificiality of the solution is the pressure required, given by (12), to maintain the wave group. Let us try to eliminate the right-hand side of (12) by taking

$$
\phi=a e^{-i k \zeta}\left(1+b_{1} \operatorname{sech}^{2} \varepsilon \xi\right) \operatorname{sech} \varepsilon \xi .
$$

Then we find that for $b_{1}=\frac{1}{4}$ we still have (10) and (11), but (12) is replaced by

$$
p_{t} / \rho=3 a \varepsilon^{2} c_{g}^{2} \operatorname{sech}^{5} \varepsilon \xi e^{-i k \zeta} .
$$


Thus the pressure distribution is more concentrated around $\xi=0$. We can go on adding terms, and the $(n+1)$-term solution is

$$
\phi=a e^{-i k \xi} \operatorname{sech} \varepsilon \xi \sum_{m=0}^{n} b_{m} \operatorname{sech}^{2 m} \varepsilon \xi
$$

where

$$
b_{0}=1, \quad b_{m}=\frac{2 m-1}{2 m+2} b_{m-1} .
$$

For the $(n+1)$-term solution given by $(17)$, the pressure distribution at the free surface is given by

$$
p_{t} / \rho=(2 n+1)(2 n+2) a b_{n} \varepsilon^{2} c_{g}^{2} \operatorname{sech}^{2 n+3} \varepsilon \xi e^{-i k \zeta},
$$

which is more and more concentrated near $\xi=0$ as $n$ increases. We will now write the real part of (19) explicitly for $y=0$ :

$$
p_{t} / \rho=(2 n+1)(2 n+2) a b_{n} \varepsilon^{2} c_{g}^{2} \operatorname{sech}^{2 n+3} \varepsilon\left(x-c_{g} t\right) \cos k(x-c t),
$$

where

$$
b_{n}=\frac{1}{4} \frac{3}{6} \frac{5}{8} \frac{7}{10} \frac{9}{12} \frac{11}{14} \cdots \frac{2 n-1}{2 n+2} .
$$

Let

$$
x^{\prime}=x-c_{g} t, \quad x-c t=x^{\prime}-\left(c-c_{g}\right) t .
$$

Then (20) becomes, for $y=0$,

$$
p_{t} / \phi=(2 n+1)(2 n+2) a b_{n} \varepsilon^{2} c_{g}^{2} \operatorname{sech}^{2 n+3} \varepsilon x^{\prime} \cos k\left[x^{\prime}-\left(c-c_{g}\right) t\right] .
$$

For large $n$ (so that $p_{t}$ is concentrated near $x^{\prime}=0$ ) integration gives

$$
p / \rho=-\frac{(2 n+1)(2 n+2)}{k\left(c-c_{q}\right)} a b_{n} \varepsilon^{2} c_{g}^{2} \operatorname{sech}^{2 n+3} \varepsilon x^{\prime} \sin k\left[x^{\prime}-\left(c-c_{g}\right) t\right] .
$$

It is interesting to see what the distribution of $p$ is for very large $n$. We note, first, that the pressure distribution becomes more and more concentrated near $x^{\prime}=0$ as $n$ increases and, second,

$$
\varepsilon \int_{0}^{\infty} \operatorname{sech}^{2 n+3} \varepsilon x^{\prime} d x^{\prime}=\int_{0}^{\pi / 2} \cos ^{2(n+1)} \theta d \theta=\frac{2 n+1}{2(n+1)} \frac{\pi}{2} c_{n},
$$

where

$$
c_{n}=\frac{2 n-1}{2 n} \frac{2 n-3}{2(n-1)} \cdots \frac{1}{2}=\frac{1}{2} \frac{3}{4} \frac{5}{6} \cdots \frac{2 n-1}{2 n},
$$

and $\theta$ is defined by

$$
\sin \theta=\tanh \varepsilon x^{\prime} .
$$


As $n$ becomes very large, $p$ is very concentrated near $x^{\prime}=0$, and (24) gives

$$
\int_{-\infty}^{\infty} p d x^{\prime}=\frac{\pi a \rho \varepsilon c_{g}^{2} \sin k\left(c-c_{g}\right) t}{k\left(c-c_{g}\right)}(2 n+1)^{2} b_{n} c_{n} .
$$

We need to evaluate $b_{n}$ and $c_{n}$ for very large $n$. Note first that

$$
\frac{1}{4} \frac{2}{5} \frac{3}{6} \frac{4}{7} \cdots \frac{2 n-3}{2 n}=\frac{6}{2 n(2 n-1)(2 n-2)}
$$

since, apart from the first three numerators of the left-hand side and the last three denominators, the rest, upon one-to-one cancelling, is equal to 1. Furthermore, it is clear that

$$
\begin{aligned}
\frac{\frac{1}{4} \frac{3}{6} \frac{5}{8} \cdots \frac{2 n-3}{2 n}}{\frac{2}{5} \frac{4}{7} \frac{6}{9} \cdots \frac{2 n-4}{2 n-1}} & =\frac{5}{2} \frac{7}{16} \frac{27}{36} \frac{55}{64} \cdots \frac{2 n-3}{2 n} \\
& =\frac{5}{2}\left(1-\frac{9}{16}\right)\left(1-\frac{9}{36}\right)\left(1-\frac{9}{64}\right) \cdots \frac{2 n-3}{2 n}
\end{aligned}
$$

which, as $n$ approaches infinity, approaches $5 Q / 2$, where

$$
Q=\left(1-\frac{9}{16}\right)\left(1-\frac{9}{36}\right)\left(1-\frac{9}{64}\right) \cdots
$$

is a convergent infinite product [3, p. 32] of value less than 1 and different from zero, since $\frac{1}{16}+\frac{1}{36}+\frac{1}{64}+\cdots$ is a convergent series. Noting that the upper product on the left-hand side of (30) is exactly $b_{n-1}$, we obtain from (29) and (30) that, for very large $n$,

$$
\frac{2}{5 Q} b_{n}^{2}=\frac{3}{4 n^{3}} \text {. }
$$

The value of $Q$ given by (31) can be determined from the well-known formula [2, p. 30]

$$
x\left(1-\frac{x^{2}}{\pi^{2}}\right)\left(1-\frac{x^{2}}{4 \pi^{2}}\right)\left(1-\frac{x^{2}}{9 \pi^{2}}\right) \cdots=\sin x,
$$

or on replacing $x$ by $3 x / 2$,

$$
\frac{3 x}{2}\left(1-\frac{9 x^{2}}{4 \pi^{2}}\right)\left(1-\frac{9 x^{2}}{16 \pi^{2}}\right)\left(1-\frac{9 x^{2}}{36 \pi^{2}}\right) \cdots=\sin \frac{3 x}{2} .
$$

If we put $x=\pi$ in this last formula, we obtain

$$
Q=\frac{8}{15 \pi} \quad \text { or } \quad \frac{5 Q}{2}=\frac{4}{3 \pi} .
$$

Thus (32) becomes, for very large $n$,

$$
b_{n}^{2}=\frac{1}{\pi n^{3}} \quad \text { or } \quad b_{n}=\left(\pi n^{3}\right)^{-1 / 2} .
$$

For the calculation of $c_{n}$ we start from the obvious formula

$$
\frac{1}{2} \frac{2}{3} \frac{3}{4} \cdots \frac{2 n-1}{2 n}=\frac{1}{2 n} \text {, }
$$


from which we obtain

$$
c_{n} d_{n}=\frac{1}{2 n},
$$

where

$$
d_{n}=\frac{2}{3} \frac{4}{5} \frac{6}{7} \cdots \frac{2 n-2}{2 n-1} .
$$

From (26) and (37) we have

$$
\frac{d_{n}}{c_{n}}=2 \frac{8}{9} \frac{24}{25} \frac{48}{49} \cdots \frac{2 n(2 n-2)}{(2 n-1)^{2}}=2\left(1-\frac{1}{9}\right)\left(1-\frac{1}{25}\right) \cdots\left(1-\frac{1}{(2 n-1)^{2}}\right)
$$

But $[2$, p. 31]

$$
\cos x=\left(1-\frac{4 x^{2}}{\pi^{2}}\right)\left(1-\frac{4 x^{2}}{9 \pi^{2}}\right)\left(1-\frac{4 x^{2}}{25 \pi^{2}}\right) \cdots
$$

and moving the first factor on the right-hand side of (39) to the other side, putting $x=\pi / 2$, and applying l'Hôpital's rule, we obtain

$$
\left(1-\frac{1}{9}\right)\left(1-\frac{1}{25}\right) \cdots=\pi / 4 \text {. }
$$

Thus, for very large $n,(38)$ and (40) give

$$
d_{n} / c_{n}=\pi / 2
$$

and from (36) we obtain, for very large $n$,

$$
\pi c_{n}^{2}=\frac{1}{n} \quad \text { or } \quad c_{n}=\left(\frac{1}{n \pi}\right)^{1 / 2} .
$$

Putting (34) and (42) into (28), we have, for very large $n$,

$$
F=\int_{-\infty}^{\infty} p d x^{\prime}=\frac{4 a \rho c_{g}^{2} \sin k\left(c-c_{g}\right) t}{k\left(c-c_{g}\right)} .
$$

Given $c_{g}$ and

$$
\omega=k\left(c-c_{g}\right),
$$

we can calculate $k$ and $c$ from (14) and (44) and then $\varepsilon$ from (13). The conclusion, then, is that if a finite force $F$ given by $4 a \rho \varepsilon c_{g}^{2} \sin \omega t / \omega$ moves with speed $c_{g}$ on the free surface, a single group of waves of wave number $k$ advances with speed $c_{g}$, whereas the individual waves advance with speed $c$. The velocity potential is given by (17). The surface displacement $\eta$ is given by (3), by integration with respect to $t$-which is not an easy task at first sight. However, at any finite distance from $x^{\prime}=0$ the pressure $p$ is zero and $\eta$ is then given by (4) to be

$$
\eta=(1 / g) \phi_{t},
$$

with $\phi$ given by (17). At $x^{\prime}=0$, it is understandable that $\eta$ is not finite. However, for a distribution of $p$ given as a function of $x, c_{g}$, and $\omega, \phi$ can be obtained by integration, and $\eta$ is then everywhere finite. 
3. Removal of singularities in the solution. It can be readily verified that the solution (6) has singularities (doublets) at

$$
z=x+i y=c_{g} t-\frac{2 N-1}{2 \varepsilon} \pi i=\alpha_{N},
$$

for any integral value of $N$ from 1 onward. The singularities can be removed by adding to (6) or (7) the terms

$$
-a e^{i k\left(c-c_{g}\right) t} \sum_{N=1}^{\infty} \frac{i(-1)^{N}}{\varepsilon\left(z-\alpha_{N}\right)} \exp \left(-\frac{2 N-1}{2 \varepsilon} k \pi\right) .
$$

Note that on the free surface

$$
\varepsilon\left|z-\alpha_{N}\right| \geqslant \frac{2 N-1}{2} \pi
$$

If

$$
\frac{k}{\varepsilon} \gg 1
$$

the magnitude of (46) is obviously extremely small at the free surface. Thus the added terms can be neglected in subsequent development up to (14).

But (15) again contains singularities. Those for the sech $\varepsilon \xi$ term have already been removed by adding (46). Those for the remaining term can be similarly dealt with.

The argument can be repeated. The singularities to be removed successively involve poles of various orders. At the $n$-th approximation the terms to be added to remove the highest singularities are of the form

$$
-a e^{i k\left(c-c_{g}\right) t} \sum_{N=1}^{\infty} \frac{(-1)^{N}(i)^{n}}{\left[\varepsilon\left(z-\alpha_{N}\right)\right]^{n}} \exp \left(-\frac{2 N-1}{2 \varepsilon} k \pi\right),
$$

and there are other terms representing poles of the first to the $(n-1)$ th orders. Again all the terms added for singularity removal have negligible effects on the calculation at the free surface, provided (48) is satisfied. Thus, with this provision, singularities can be removed from (17) for any $n$ to produce a nonsingular solution that leaves intact (13), (14), and (43), which constitute the main conclusions of this paper.

Acknowledgment. This work has been sponsored by the Fluid Mechanics Program of the Office of Naval Research.

\section{REFERENCES}

[1] H. Lamb, Hydrodynamics, Dover, New York, 1945

[2] G. Polya, Induction and analogy in mathematics, Princeton Univ. Press, Princeton, N. J., 1954

[3] E. T. Whittaker and G. N. Watson, Modern analysis, Macmillan, New York, 1945 